$\underline{\text { Penultimate draft. For published version see: }}$

https://onlinelibrary.wiley.com/doi/10.1111/mila.12358

\title{
Spatial content of painful sensations
}

\section{Błażej Skrzypulec}

Institute of Philosophy, Jagiellonian University, Kraków, Poland

Philosophical considerations regarding experiential spatial content have focused on exteroceptive sensations presenting external entities, and not on interoceptive experiences that present states of our own body. A notable example is studies on interoceptive touch, in which it is argued that interoceptive tactile experiences have rich spatial content such that tactile sensations are experienced as located in a spatial field. This paper investigates whether a similarly rich spatial content can be attributed to experiences of acute, cutaneous pain. It is argued that such experiences of pain do not have field-like content, as they do not present distance relations between painful sensations.

\section{KEYWORDS}

bodily sensations, interoception, pain, perception, touch, spatial content

\section{INTRODUCTION}

Normal sensory experiences not only present some entities as having properties, but also present them as positioned in some type of space. In representationalist terms, it can be stated that such experiences have some spatial content in virtue of which entities can be presented as 
spatially located, as occupying distinct places, or as standing in spatial relations to each other. Visual experiences provide a paradigmatic example, as in the case of vision, objects are experienced as positioned in a bounded, topologically connected spatial field and as standing in a variety of egocentric and allocentric spatial relations (see Mac Cumhaill, 2015; Martin, 1993; Richardson, 2010 for considerations about spatial aspects of vision). However, it is far from obvious that all sensory experiences have the same spatial content as normal visual experiences. In fact, one can observe a growing interest in investigations concerning the spatial content of non-visual sensory experiences. In particular, philosophers of perception have developed competing accounts of olfactory (see Batty, 2010; Millar, 2019; Richardson, 2013; Young, 2020) and auditory (see Nudds, 2009; O'Callaghan, 2008; O’Shaughnessy, 2009) abilities for spatial representations. For instance, it is discussed whether odors can be olfactorily represented as positioned in some direction from the perceiver (e.g., Aasen, 2019) or whether we have the ability to auditorily represent empty spaces (e.g., Young, 2017).

Nevertheless, such investigations are mainly concerned with exteroceptive experiences, that is ones which present external entities, and not with interoceptive experiences, which present states of our own body. ${ }^{1}$ A notable exception is constituted by studies concerning tactile modality, which is often characterized as having a dual exteroceptive and interoceptive character (e.g., Fulkerson, 2011; Mattens, 2016; Ratcliffe, 2008; Richardson, 2011). A tactile experience usually has an exteroceptive component, in which some properties are attributed to an external entity (e.g., it may be presented that a held object is round) and an interoceptive component presenting a state of one's body (e.g., that pressure is applied to a certain bodily location). Contemporary empirical studies suggest that

\footnotetext{
${ }^{1}$ In some works the term "interoceptive states" is used in a narrower way, referring only to visceral sensations (see Ceunen et al., 2016; Ferentzi et al., 2018 for a discussion). However, here I adapt a broader understanding which encompasses all experiences presenting bodily states.
} 
in case of interoceptive touch we are not only able to experience simultaneous tactile sensations as positioned in distinct bodily places (Mancini et al., 2014), but also to experience distance (Longo \& Golubova, 2017), direction (Haggard \& Giovagnoli, 2011), and topological relations between them (Fardo et al., 2018). Furthermore, it has been proposed that these spatial abilities of interoceptive touch may arise from a low-level bodily representation known as "skin-space" determined by the array of receptive fields of cutaneous tactile receptors (Cheng \& Haggard, 2018; Haggard et al., 2017). It is argued that, in virtue of skin-space, tactile sensations are experienced as located in a tactile field which, by analogy with the visual field, is a topologically connected space allowing for experience of a variety of spatial relations between presented elements (Cheng, 2019; Haggard \& Giovagnoli, 2011). If this is true, then interoceptive touch can provide experiences with rich spatial content without relying on sensory information provided by other senses such as proprioception or vision.

In the paper, I investigate, relying on the empirical state of the art, the spatial content of another major type of interoceptive states: experiences of pain. In particular, my goal is to establish whether painful sensations also have a rich spatial content analogous to interoceptive tactile experiences ${ }^{2}$ even without being supplemented with information from different modalities like touch or proprioception. I argue for a negative thesis, that the spatial content of experiences of pain is less developed than that of interoceptive tactile experiences. Most importantly, there are data suggesting that we are not presented with distance relations between painful sensations (Mancini et al., 2015). In consequence, while there may be good reasons to postulate that interoceptive touch presents sensations as positioned in a bodily tactile field, postulating an analogous pain field is not justified given the current state of knowledge.

\footnotetext{
${ }^{2}$ By "interoceptive tactile experiences" I mean interoceptive aspects of tactile experiences which, as stated earlier, in many cases also have some exteroceptive aspects.
} 
It should be noted that my argument is consistent with a thesis that in everyday situations painful sensations have multimodal spatial content, created by using information provided by proprioception, touch, and other sensory systems, which may be described in terms of a spatial field. However, I argue that empirical investigations suggest that unimodal spatial content of pain experiences, without supplementation from other systems, is not sufficiently developed to justify postulating a field-like spatial representation. This differentiates experiences of pain from interoceptive tactile experiences, where there is strong evidence that they possess rich, unimodal spatial field-like content. On the other hand, my investigations also demonstrate that, even without support from touch and proprioception, experiences of pain possess a certain, more rudimentary form of spatial content. In consequence, while there is probably no unimodal spatial pain field, it is also not the case that spatiality of pains is essentially multimodal such that without input from touch or proprioception pains are not spatial at all.

In philosophical studies on pain it is often proposed that experiences of pain have two aspects: a descriptive aspect that determines the qualitative characteristics of pain, and an evaluative aspect that determines the unpleasantness of painful experiences (e.g., Bain, 2013; 2014; Bain \& Brady, 2014; Corns, 2018; Nelkin, 1994; de Vignemont \& Jacob, 2012). While philosophers frequently claim that pains are experienced as being located within the body, as having spatial extension, and as having a spatial part-structure (see Gozzano, 2019; Hill, 2004; Smith, 2011; de Vignemont, 2017), there are no detailed investigations concerning the spatial contents of experiences of pain. The majority of philosophical works on pain concern the perceptual status of painful experiences (e.g., Aydede, 2017; 2018; Bain, 2003; Corns, 2014; Gray, 2018), the nature of evaluative aspect of pains (e.g., Cutter \& Tye, 2011; Gray, 2014; Helm 2012; Klein, 2012; O’Sullivan \& Schroer, 2012; Rachels, 2000), or a metaphysical question on whether pains are mental elements or physical entities, like certain 
disorders, positioned within the body (e.g., Bain, 2007; Holly, 1986; Hyman, 2003; Reuter, 2017; Tye, 2002). In my investigations, I attempt to stay neutral on these issues, as the only substantial assumption I make is that experiences of pain have some content specifying the experienced location of a painful sensation. Furthermore, I restrict my investigations to acute, cutaneous pains that seem to occur at the bodily surface, as they are most likely to have a rich spatial content, especially in comparison to deep, visceral or muscle pains and chronic pains (see Borg et al., 2020; Haggard et al., 2013; Leder, 2016; Svensson \& Avendt-Nielsen, 1995).

The paper starts (Section 2) by presenting different types of spatial content that jointly constitute field-like content. Subsequently, in Section 3, I present results suggesting that interoceptive tactile experiences possess field-like content. Finally, in Section 4, I consider whether analogous evidence is available in case of experiences of pain. I argue, relying on studies by Mancini et al. (2015), that experiences of pain do not have content that allows for the presentation of distance relations, and so they do not have field-like content.

\section{VARIETY OF SPATIAL CONTENT}

While most sensory experiences seem to have some spatial content, the specific characteristics of such content may be different in experiences belonging to distinct types. Thus, in order to assess the spatial content of experiences of pain and how it differs from the content of interoceptive tactile experiences, one must specify the possible variations between sensory spatial contents.

The most rudimentary form of spatial content has been described by Austen Clark (2004) using the notion of "feature-placing." A sensory modality which has the ability for feature-placing can present that some elements are positioned in some locations. For instance, it can be presented that there is a burning pain in location $L$ and an intense pain in location $P$. 
Nevertheless, an experience merely having feature-placing content is not able to present anything beyond the fact that there are some elements in some locations. In particular, it is not able to present whether the locations in which elements are positioned are identical or not. Referring to the previous example, if experiences of pain have only feature-placing spatial content, then a situation in which a burning pain and an intense pain are in the same location is not presented differently from a situation in which the locations of a burning pain and an intense pain are distinct. It is so because experiences with pure feature-placing content have no ability to present whether location $\mathrm{L}$ is identical to location P. In other words, featureplacing content can be described by using a language that has names for presented elements, names for presented locations, and a two-place predicate referring to a placing-relation that connects elements with locations. However, such a language is not equipped with the identity predicate.

A richer type of experiential spatial content is "identity content," which makes it possible to present elements as positioned in the same or distinct locations. In opposition to feature-placing content, such content cannot be described by using a language without the identity predicate. If experiences of pain possess identity content, then painful experiences are not only able to present that pain $F$ is positioned in location $L$ and pain $G$ is positioned in location $P$, but also are able to present that $L$ and $P$ are distinct. Nevertheless, the identity content is still quite rudimentary, as experiences having only such spatial content cannot present the spatial relations between locations, and so cannot present the structure of the space in which some elements are experienced as located.

In consequence, richer variants of spatial content may be obtained by extending identity content such that it can characterize not only identity but also various spatial relations between locations. One type of such richer spatial content is "distance content," which can specify not only that locations $\mathrm{L}$ and $\mathrm{P}$ are distinct, but also that they stand in some distance 
relation D. Another is "directional content," due to which elements are presented as located in places positioned in some direction from one another or from the subject perceiving them. Finally, one can distinguish "topological content," in virtue of which it may be presented that certain locations are spatially connected, that is their borders are in contact or they partially overlap. Such content is crucial for being able to present that the space of perceived elements is topologically connected in the sense that for each pair of locations within the space there is a continuous path composed of locations also belonging to the considered space.

If an experience possesses all of these types of spatial content (feature-placing content, identity content, distance content, directional content, and topological content), then it can be stated that such an experience has a field-like spatial content, because it presents entities as positioned within a spatial field. A perceptual spatial field is often characterized by using a metaphor of a "container" or "form" (see Cheng, 2019), which suggests a topologically connected space such that entities positioned within it can be further from or closer to each other and may be positioned in various directions. In fact, one of the most common arguments in favor of attributing field-like content to tactile experiences concerns the presence of results demonstrating that tactile perception is able to compute spatial relations between bodily sensations (see Cheng \& Haggard, 2018, pp. 62-63; Fardo et al., 2018, p. 101; Haggard \& Giovagnoli, 2011). It is intuitively plausible to ascribe field-like content to usual visual experiences that present objects as located in a topologically connected visual field and standing in a variety of egocentric and allocentric distance and direction relations. However, it is debated whether other sensory modalities also present entities as located in some form of spatial field (see Martin, 1993; Phillips, 2013; Scott, 2001; Soteriou, 2011; Young, 2020).

It should be noted that it not necessarily the case that all experiences with field-like content present space in exactly the same way. In particular, some experiences may present spatial relations using an allocentric frame of reference, while other may utilize an egocentric 
or a somatotopic framework. For instance, while visually presented distance relations may characterize how far an object is from a perceiver (see Alsmith, 2017), the interoceptive tactile distance relations are likely to consider the amount of skin surface separating felt sensations (see Haggard \& Giovagnoli, 2011; Longo \& Golubova, 2017). Furthermore, sensory spatial fields can differ in shape in a manner determined by their topological structure. For example, the human visual field is usually characterized as having a threedimensional conical shape (see Richardson, 2010) while the interoceptive tactile field is conceptualized as a two-dimensional matrix determined by an array of skin receptors (see Cheng \& Haggard, 2018; Haggard et al., 2017). Experiences with the same type of spatial content may also differ in quantitative aspects regarding the precision of their representations. For instance, while both visual and auditory experiences plausibly have distance content, it is likely that audition presents the distance of entities less precisely than vision. Similarly, while visual and auditory experiences can present distance relations, it is likely that they cannot present any distance, but only distances within a certain range.

In the next section, I present evidence for a thesis that interoceptive tactile experiences have field-like content. In doing so, I do not postulate that the current state of the art provides a definitive argument for tactile field-like content. However, I show that there are important data suggesting attributing rich spatial content to interoceptive tactile experiences. Furthermore, I do not argue that all interoceptive tactile experiences have such rich content, but I present arguments for a weaker thesis, that field-like content can be attributed to many of the usual interoceptive tactile experiences. These considerations allow further investigation of whether analogous evidence is available in the case of experiences of acute, cutaneous pain.

\section{INTEROCEPTIVE TOUCH AND SPATIAL CONTENT}


By interoceptive touch I mean those aspects of tactile modality which present states of one's body, for instance, that there is a pressure inflicted on a bodily surface. One of the most important paradigms in studies regarding spatial aspects of interoceptive touch consists of measuring the two-point-distance (e.g., Haggard \& Giovagnoli, 2011; Haggard et al., 2017; Mancini et al., 2013). In such studies, two tactile stimuli are simultaneously applied to a fragment of the bodily surface and the task of a participant is to state whether she felt one tactile sensation or two spatially separated sensations. The two-point-distance is the minimal distance that allows the participant to recognize that two stimuli were used; applying this procedure to various bodily parts allows researchers to establish how the spatial discriminatory abilities of interoceptive touch change across the body (see Mancini et al., 2014). Of course, to avoid recognizing the distinction between applied stimuli by some additional, nonspatial means, other properties of stimuli, such as size or pressure, are the same, and participants do not have visual access to the stimulated bodily fragment.

The simple fact that people can recognize, given sufficient spatial separation, that two, and not one, stimuli have been simultaneously applied already shows that interoceptive tactile experience possesses not only feature-placing content but identity content. An experience with mere feature-placing content can only present there is a sensation at a place $\mathrm{P}$ and that there is a sensation at a place $\mathrm{L}$ but cannot determine whether $\mathrm{L}=\mathrm{P}$, and so whether what was experienced was two spatially distinct sensations. This additional ability is possessed by experiences with identity content that allow determination of the sameness of locations occupied by presented elements. Nevertheless, while two-point-distance studies demonstrate the presence of interoceptive tactile identity content, they do not allow ascription of any richer spatial content to tactile experiences, as the task does not require recognition of any spatial relations beyond mere non-identity. 
Nevertheless, studies on the spatial abilities of interoceptive touch are by no means restricted to measuring two-point-distance, and there are empirical data suggesting that interoceptive tactile experience have richer spatial content than merely identity content. For instance, there are studies in which two spatially disjoint stimuli are simultaneously or diachronically applied to a bodily fragment, but the task is not to state whether one or two sensations were felt, but to estimate the distance between sensations (see Haggard et al., 2017; Longo \& Golubova, 2017; Mancini et al. 2015; de Vignemont et al., 2009). While the judgments participants provide are not without inaccuracies and biases, statistical analysis of the data obtained reveal a general, linear pattern such that when stimuli are physically further apart (that is are separated by a larger skin-fragment) the corresponding tactile sensations are also judged to be divided by a greater distance. The ability to distinguish between tactile sensations positioned closer to and further from each other shows that interoceptive tactile experiences have also distance content, which allows the perception of spatial relations of distance.

Furthermore, there are also results that suggest the presence of directional content in interceptive tactile experiences. In particular, the ability to discriminate directional relationships between tactile sensations has been tested by Haggard and Giovagnoli (2011). Their experimental design consisted of two steps. First, tactile stimulation was applied on a hand or forearm, designating a line which served as a reference point for further directional judgments. Subsequently, a target stimulus occurred on one side of the designated line, and the task of the participant was to state whether a sensation was felt on the right or on the left of the line. Successful performance in such a task cannot be explained by mere presence of identity content, as the question is not simply to decide whether the target sensation is positioned outside the designated line. It also cannot be explained by the presence of distance content, as the distance of the target stimulus from the line was the same no matter the 
direction. In consequence, it seems that interoceptive tactile experiences have both distance and directional content.

Finally, the presence of topological content is justified by referring to the results of tactile path integration studies (Fardo et al., 2018). In such studies, a tactile stimulus is continuously moved across a fragment of the skin along a curved, S-shaped path. When the stimulation ends, the participant is asked to point out the middle location between the starting and the ending point of stimulation. Because the stimulus creates a curved path on the skin, the middle point along the shortest path between starting and ending position does not receive any tactile stimulation. Success in this path integration task shows that interoceptive tactile experiences have quite rich spatial content. In particular, to recognize the middle location between starting and ending point of stimulation, it has to be presented that starting and ending points are distinct, and it has to be presented that they are at some distance from one another in order to identify a location that is equidistant from both points. However, recognizing a middle point that has not been stimulated requires not only presenting that tactile sensations were positioned at some distance and direction from each other, but also that there is some space extending between sensations. This suggests some form of topological content, presenting that there is a continuous path between the starting and the ending point on which the middle, target point lies.

The studies discussed here suggest that interoceptive tactile experiences have field-like spatial content in virtue of having feature-placing, identity, distance, directional, and topological content. However, one may doubt whether the field-like content considered is actually tactile content and not a multimodal content which combines tactile cutaneous information with, inter alia, proprioceptive information concerning the actual position of bodily parts (e.g., Carruthers, 2008; Lederman \& Klatzky, 2009; Proske \& Gandevia, 2012) and information about stable bodily structure stored in long-term bodily representations such 
as "off-line body schema" (e.g., Hochstetter, 2016; Longo \& Haggard, 2010; de Vignemont, 2010) or "superficial schema" (see Head \& Holmes, 1911 for a classic source and Longo, 2010 for a contemporary review). Nevertheless, I believe that there are certain reasons that, while not definitive, make it plausible to accept that the described field-like content is genuinely tactile.

In particular, the most of the described studies do not explicitly require utilizing data provided by non-tactile means. For instance, two-point-distance tasks do not require the participant to recognize on which body part the sensations are localized or whether each sensation is on the same body part. Such additional abilities are likely to demand further information regarding how the body is divided into parts by joints and how the bodily parts are currently positioned (see de Vignemont, 2014). Similarly, the experimental tasks aimed to investigate tactile interoceptive distance perception do not require recognizing on which bodily parts sensations are positioned or judging their distance from some bodily landmark, like a wrist or elbow. While determining the distance from a bodily landmark is likely to require a combination of tactile and proprioceptive information, the mere assessment of distance between sensations within somatotopic space can be plausibly made by relying on a specific pattern of cutaneous receptors that are stimulated vs. not stimulated. For instance, it has been proposed that the distance between sensations can be computed by a mechanism which recognized the number of non-stimulated receptive fields of tactile receptors separating the stimulated receptive fields (see Cheng \& Haggard, 2018; Haggard et al., 2017).

Of course, this is not to state that proprioceptive information concerning division of body into parts does not influence how distance relations between tactile sensations are experienced. In particular, it has been discovered that the perceived distance between sensations is enlarged when each sensation is positioned on a different body part (for instance, they are separated by a joint, see Knight et al., 2014; de Vignemont et al., 2009). However, 
the presence of such influences does not justify a thesis that there is no ability for experiencing distance relations between tactile sensations relying solely on cutaneous data. In particular, adaptation aftereffects concerning interoceptive touch have been obtained that are confined to a stimulated skin region and do not transfer contralaterally. The presence of such aftereffects suggests that interoceptive tactile distance is already computed at early stages of sensory processing relying on local, cutaneous data (see Calzolari et al., 2017). The situation is more complicated in case of studies on directional discrimination, as they have explicitly asked whether a sensation is positioned to the left or right of the established tactile line (Haggard \& Giovagnoli, 2011). Making such discrimination requires representing bodily parts as positioned within an egocentric framework relying on data concerning bodily partstructure. However, it seems plausible that participants would still be able to succeed in the experimental task even if they were not asked to determine "left" or "right" direction but, for instance, state whether an actual sensation is on the same side of the line as the previous sensation or not. Nevertheless, further studies are needed to investigate this idea.

Furthermore, there are important theoretical accounts which explain how the field-like content can be obtained by relying on cutaneous tactile perception. In particular, a notion of "skin-space" has been proposed which is a bodily representation representing the surface of the body relying on the array of cutaneous receptive fields (Cheng, 2019; Cheng \& Haggard, 2018; Haggard et al., 2017, see also Longo \& Golubova, 2017 for a conceptualization in terms of a 2D array of pixels). Such a representation allows interoceptive tactile experiences to have identity content, as when stimuli are spatially disjoint, there are parts of the skin-space in which stimulation is present separated by a part that lacks stimulation. Furthermore, topological content is supported by the fact that there is a continuous path between each two locations within the skin-space determined by neighboring or overlapping receptive fields of cutaneous receptors. In consequence, distance relations can be represented, as nearby 
sensations are divided by a higher number of receptive fields than further ones. Similarly, directional content can be obtained by relying on the fact that if tactile sensations A and B lie in distinct directions from a certain sensation $\mathrm{C}$, then the shortest paths in skin-space from $\mathrm{C}$ to A and from C to B do not overlap. Of course, the structure of skin-space does not guarantee that spatial relations are represented without biases and inaccuracies. For instance, as the size of cutaneous receptive fields changes across the body, representing distance relations by counting receptive fields between sensations may lead to overestimation or underestimation of distance depending on which skin fragments were stimulated (see Matthen, forthcoming). Nevertheless, despite such possible inaccuracies, skin-space may serve as a foundation of qualitative ability for presenting spatial relations relying on cutaneous information.

My goal is not to argue that the above considerations definitively prove that interoceptive tactile experiences have a field-like spatial content. However, they show that there is important evidence supporting such a claim. In the next section, I consider whether analogously strong evidence is present in case of spatial content of experiences of pain.

\section{SPATIALITY OF PAINS}

In this investigation concerning the spatial content of experiences of pain I focus on acute,

cutaneous painful sensations. Similarly, as in case of interoceptive tactile experiences, I am interested whether usual experiences of acute pain have rich, field-like content without significantly relying on spatial information provided by different sensory modalities such as proprioception, touch, or vision (see De Paepe et al., 2014; Gallace et al., 2011, Marotta et al., 2015 for studies showing how proprioceptive information modifies experiences of pain). Intuitively, it may seem that the spatial content of experiences of cutaneous pain should be similar to the spatial content of interoceptive tactile experiences, as in addition to tactile skin- 
space, there may be a nociceptive skin-space determined by the receptive fields of appropriate receptors (see Koltzenburg et al., 1993) and supported by the presence of detailed cortical nociceptive maps (see Mancini et al., 2012). Nevertheless, the mere description of representational vehicle structures does not allow inference of the content of painful experiences, as the content depends in an important way on how the nociceptive information is computed. Further, I argue that it is unjustified to attribute field-like content to experiences of pain as there are results strongly suggesting that such experiences lack distance content.

In contemporary experiments, the most common method of studying cutaneous, acute pain is to apply a laser stimulus to the skin surface to produce a brief sensation of "pinprick pain." This method of stimulation has two main advantages: First, it makes possible to evoke a painful sensation without simultaneously applying a tactile stimulus; second, the use of laser allows obtaining high precision in selecting the stimulated skin fragment. It seems intuitively obvious that pains are experienced as having bodily locations. In fact, a number of empirical results have been obtained that show that cutaneous painful sensations can be localized at least as precisely as tactile sensations (e.g., Haggard et al., 2013; Koltzenburg et al., 1993; Moore \& Shady, 1995; Schelreth et al., 2001). Such results are obtained even if surface pain is evoked without simultaneous tactile processing by using a laser stimulus or by mechanically blocking tactile afferent fibers. Nevertheless, the fact that we feel pains as positioned on the body's surface, and we can locate them with high precision, does not show anything beyond the fact that experiences of pain have some feature-placing content. Furthermore, study designs in which participants are asked to point out the bodily location of a painful sensation involve a strong proprioceptive spatial component as to succeed in such task a spatial relation between the painful fragment of the body and a finger or other pointing device has to be recognized. 
Despite the above limitations, there are also studies which more directly show that experiences of pain have both feature-placing and identity content. Such works rely on the two-point-distance paradigm, analogous to that used in studies on touch described in the previous section, and apply it to the research on pain (Mancini et al., 2014; 2015). These investigations show that, similar to the case of interoceptive touch, people can differentiate situations in which one painful stimulus was applied from cases in which two spatially separated stimuli were used. In fact, it has been observed that, on certain bodily parts, for instance the abdomen, the two-point-distance is smaller for painful sensations than for tactile sensations. This means that there are some distances at which two tactile stimuli are indistinguishable from a single stimulus, but painful stimuli are still recognized as distinct. In the majority of contemporary studies, the painful sensations are evoked by laser stimulation, which allows avoiding simultaneous activation of the tactile system. Thus, each such stimulation causes a qualitatively similar "pinprick pain" and so two simultaneously applied stimuli must be distinguished by a difference in location and not by other qualitative characteristics. Furthermore, as in tactile two-point-distance studies, participants are not required to identify where the sensations are positioned within the bodily structure but simply to state whether one or two sensations are present. It makes it more plausible that featureplacing and identity spatial content of painful experiences are not dependent on utilizing some additional, for instance proprioceptive, sensory information, or at least that such an independence thesis has equally strong support as is available for interoceptive touch.

A clear picture is harder to obtain in case of relational spatial content of experiences of pain. In particular, there are no data on pain analogous to those provided by tactile path integration studies. Thus, it is difficult to assess whether painful sensations have topological content. Furthermore, while there are studies showing that directional discrimination between painful sensations is possible, the design of these studies is such that the successful resolution 
of the experimental task substantially relies on utilizing additional information regarding bodily structure. In these studies (Mancini et al., 2013; 2014, see also Schelreth et al., 2001), two painful sensations are evoked one after another by applying laser stimuli to a bodily fragment. The task is to recognize whether the second stimulus was more proximal (e.g., closer to the shoulder) or more distal (e.g., closer to the palm) from the first. While making such judgments requires recognizing differences in direction between the locations of painful sensations, the considered directions are defined in reference to the bodily structure, and so recognizing them requires the use of information related to proprioception and bodily representations, such as body schema. A similar problem has been also present in investigations regarding directional relations between interoceptive tactile sensations that asked whether a tactile sensation was positioned on the left or right of a predetermined tactile line (Haggard \& Giovagnoli, 2011). In the case of painful sensations, it seems quite plausible that a success rate would also be high in a modified paradigm that does not require referring to a proximal/distal distinction, but in which, for instance, participants are asked whether successively evoked painful sensation are in the same or in a different direction from some nociceptively pre-determined place on the skin. However, further data would be required to confirm this hypothesis.

Of course, the lack of straightforward evidence in favor of topological and directional content for painful sensations does not equal the presence of evidence against the thesis that painful sensations have such spatial content. However, in the subsequent paragraphs I argue that negative evidence is available regarding distance content, as there are data which strongly suggest that spatial relations concerning distance between painful sensations are not presented.

The crucial study regarding distance relations and experiences of pain was conducted by Mancini and colleagues (Mancini et al., 2015). As in investigations concerning 
interoceptive tactile distance content, the experimental task was to assess the distance, in centimeters, between simultaneously evoked sensations. Both tactile and laser stimuli were used to allow direct comparison of participants' abilities to make distance judgments regarding painful sensations obtained without stimulation of tactile system and non-painful tactile sensations. The task was preceded by measuring the two-point-distance to ensure that the distance between sensations was sufficient to allow them to be easily recognized as two spatially distinct sensations. The physical distances used ranged between $120 \%$ to $300 \%$ of the two-point distance, and stimuli were applied to the palm or abdomen. The data from each body location of each participant were used to create a regression model describing how assessments of the perceived distance between sensations are correlated with changes in physical distances between stimulated skin locations.

In the case of non-painful tactile stimulation, the expected results were obtained: data from all participants, gathered from both palm and abdomen, showed a clear correlation between physical and perceived distance: greater physical distances corresponded to greater perceived distances. Furthermore, for all participants, the constructed regression models were significant - that is, the correlations between perceived and physical distance allowed confident rejection of the null hypothesis that there is no linear relationship between changes in physical distance between stimuli and perceived changes regarding distance between sensations.

The results were radically different for painful sensations. For the most of the participants, no matter the bodily location, the regression models were not significant. In other words, the results did not allow the researchers to reliably reject the null hypothesis that changes in physical distance are not linearly correlated with assessments of the perceived distance. Furthermore, while in cases of tactile stimulation, greater physical distances always correlated with greater perceived distances, all possible patterns were observed when painful 
sensations were concerned: in some participants, greater physical distance corresponded to greater perceived distances; in some participants, perceived distances were virtually constant despite changes in physical distances; and in some participants, the correlation was negative such that greater physical distance corresponded to smaller perceived distances. In addition, the researchers conducted an experiment to ensure that these results did not arise as a result of low ability to localize painful sensations when two such sensations are present simultaneously (Mancini et al., 2015, p. 43). However, no significant differences concerning the accuracy of localization were found between situations in which one and two painful sensations were evoked.

These differences in distance assessments between tactile and painful sensations can be interpreted in two major ways. First, a weaker, quantitative interpretation states that distance relations are presented both between tactile and painful sensations, but that the distance content is less precise for painful sensations. Second, a stronger, qualitative interpretation postulates that, in opposition to interoceptive tactile experiences, experiences of cutaneous pain do not have distance content. Below, I argue that the stronger interpretation is more plausible.

One may have an intuition that the mere fact that people are able to provide a judgment regarding the distance between painful sensations is sufficient to justify that there is some ability to present spatial distance relations. If such ability were not present, people would not be able to provide any assessment of distance. Nevertheless, this argument is unsuccessful for two reasons. First, the mere presence of identity content allows recognition that locations of sensations are distinct, and so the distance between them is larger than zero. Second, the general knowledge of the bodily size and structure allow inference that distances between sensations on a given part of body must be within a certain range. For instance, it would be unreasonable to judge that that distance between sensations on the palm of a hand is 
50 centimeters. In consequence, even if experiences of pain do not have any distance content, a person has additional information which allows production of a judgment expressing a nonzero distance located within a certain range.

A different idea is to claim that results of Mancini et al. (2015) do not show that there is no distance content of painful sensations, but that the assessments of distance are simply heavily biased. Such a bias may be linear, leading all assessments of perceived distance to be overestimated or underestimated. However, the presence of a linear bias is not supported by the results presented. A linear bias would change slopes of functions describing the relationship between perceived and physical distance, but it would also result in a coherent relationship between physical distance and perceived distance. However, the results do not show a coherent relationship of this type; for the majority of participants, the regression model was not significant and various correlations, both positive and negative, were observed between physical and perceived distance. Of course, a bias in recognizing the distance between painful sensations could also be nonlinear. For instance, it may be the case that small and large distances are overestimated while medium distances are underestimated. Nevertheless, Mancini et al. explicitly claim that they were searching for nonlinear relationships but did not find any (Mancini et al., 2015, pp. 44-45).

A proponent of the weaker, quantitative thesis may claim that the considered results are not produced by a systematic bias, but rather by random inaccuracies. The assessments of distance are sometimes overestimated and sometimes underestimated without any salient regularity. Such an idea may be plausible only if there is some pattern in the data that shows a genuine ability to recognize distance despite the presence of inaccuracies. Otherwise, even a completely random set of judgments could be interpreted as showing an ability to recognize distance relations, as in principle any random set of responses can arise due to some specific pattern of inaccuracies. I believe that a minimal requirement for ascribing the genuine ability 
of presenting distance relations to some sensory system is that such a system demonstrates a reliable ability to make qualitative distance differentiations, that is it is able to discriminate larger distances from shorter distances. Without such an ability it would be difficult to state that what such a system presents is distance and not some other property. On the other hand, the ability for making such qualitative discriminations is coherent with presence of some biases and inaccuracies. Nevertheless, the results of Mancini et al. (2015) do not provide support for the claim that such a qualitative ability is present in the case of painful sensations. For the vast majority of participants, the regression models were not significant no matter the bodily location. This means that the obtained data do not allow the authors to reliably refute the null hypothesis that changes in distance judgments are not linearly correlated with changes in physical distance. However, refuting the null hypothesis is necessary to justify the claim that there is an ability to make qualitative distance discriminations in experiences of pain. The ascription of such ability assumes a linear relationship such that shorter physical distances are judged as shorter than longer physical distances.

The Mancini et al. (2015) results provide strong support for a thesis that experiences of pain do not have distance content. In consequence, it is plausible that they do not have field-like content, as having distance content is a necessary condition of having field-like content. Thus, it is likely that the spatial content of experience of pain is less developed than the spatial content of interoceptive tactile experiences; for the latter, there are data suggesting the presence of all relevant types of spatial content. It seems that interoceptive experiences can significantly differ in their spatial content even if, like interoceptive tactile experiences and cutaneous pains, they are experienced as occurring in the same places, on the boundaries of the body. Furthermore (see Cheng, 2020 for this observation), lack of rich spatial content may be one of the reasons why experiences of pain, in contrast to tactile experiences, do not have both interoceptive and exteroceptive aspects, but are purely interoceptive. This is 
because the ability to represent spatial relations between cutaneous stimuli may be required to ascribe spatial properties to external entities causally affecting the skin.

A probable hypothesis is that to obtain distance content, the content of the experiences of pain must be supplemented by content provided by proprioception and structural bodily representations. In particular, participants in the study of Mancini et al. (2013) were able to state which painful sensation is more proximal or more distal in relation to the bodily structure. In this case, the presented distance does not concern spatial relations between painful sensations, but between a painful sensation and some distinguished bodily fragment. In consequence, the results of Mancini et al. (2013) demonstrate an ability to make a qualitative discrimination between longer and shorter distances involving a painful sensation. However, such distance assessments do not rely merely on cutaneous nociceptive information, but require information regarding the organization of the body into parts connected by joints.

Of course, as stated in the introduction, usual experiences of pain are likely to have a multimodal spatial content resulting from information provided by various sensory systems. The impact of these sensory systems on spatial content of pains and whether, in virtue of such influence, a field-like content is obtained are matters for further investigations, going beyond the scope of this paper, which focuses on unimodal spatial content of experiences of pain and demonstrates that such content itself is unlikely to be field-like content. On the other hand, there is strong evidence that experiences of pain, even without support from additional systems, have feature-placing and identity contents, so unimodal content of pain experiences is not entirely without spatial character. Furthermore, it should be noted that even in considerations regarding unimodal spatial content of pains the current state of knowledge does not allow for its full characterization. While study by Mancini et al. (2015) provides good reasons for rejecting distance content of experiences of pain, distance relations are not the only ones that can be presented by sensory experiences. In fact, there are currently no data 
which would allow formulation of any decisive thesis regarding topological content and the ability to present directional relations between painful sensations independently from additional (e.g., proprioceptive) information. Further studies may establish whether they are also able to present topological relations, directional relations, or both.

\section{CONCLUSIONS}

While it is common for sensory experiences to have spatial content, the spatial characteristics of interoceptive experiences have attracted less philosophical attention than the spatial characteristics of exteroceptive experiences. Recently, it has been argued that interoceptive tactile experiences, even without help from different sensory systems, may have rich spatial content, similar in important aspects to visual spatial content. In technical terms, it can be stated that both visual and interoceptive tactile experiences have field-like spatial content in virtue of having feature-placing, identity, distance, directional, and topological contents. A question arises whether other types of interoceptive experiences, such as cutaneous, acute pains, have similarly rich spatial content. Though investigations concerning spatiality of pain are less developed than those of tactile experiences, there are important data suggesting that experiences of pain do not have distance content. In consequence, they do not have field-like content despite being experienced as positioned on skin surface in a manner analogous to interoceptive tactile sensations. Further investigations, for instance concerning thermal perception (see Ferrè et al., 2018; Marotta et al., 2015), may reveal whether conclusions regarding spatial content of cutaneous pains are also applicable to other types of interoceptive experiences.

\section{ACKNOWLEDGEMENTS}


The author would like to thank the anonymous reviewers for their comments.

\section{REFERENCES}

Aasen, S. (2019). Spatial aspects of olfactory experience. Canadian Journal of Philosophy, 49(8), 1041-1061.

Alsmith, A. J. T. (2017). Perspectival structure and agentive self-location. In De Vignemont, F., \& Alsmith, A., (Eds.), The subject's matter: Self-consciousness and the body (pp. 263288). Cambridge, MA: MIT Press.

Aydede, M. (2017). Pain: Perception or introspection? In Corns, J., (Ed.), Routledge handbook of philosophy of pain (pp. 221-232). London/New York, NY: Routledge.

Aydede, M. (2018). A contemporary account of sensory pleasure. In Shapiro, L., (Ed.), Pleasure: A history (pp. 239-266). Oxford: Oxford University Press.

Bain, D. (2003). Intentionalism and pain. The Philosophical Quarterly, 53(213), 502-523.

Bain, D. (2007). The location of pains. Philosophical Papers, 36(2), 171-205.

Bain, D. (2013). What makes pains unpleasant?. Philosophical Studies, 166, Supplement 1, 69-89.

Bain, D. (2014). Pains that don't hurt. Australasian Journal of Philosophy, 92(2), 305-320.

Bain, D., \& Brady, M. (2014). Pain, pleasure, and unpleasure. Review of Philosophy and Psychology, 5, 1-14.

Batty, C. (2010). A representational account of olfactory experience. Canadian Journal of Philosophy, 40(4), 511-538.

Borg, E., Harrison, R., Stazicker, J., \& Salomons, T. (2020). Is the folk concept of pain polyeidic?. Mind \& Language, 35(1), 29-47. 
Ceunen, E., Vlaeyen, J. W. S. \& Van Diest, I. (2016). On the origin of interoception. Frontiers in Psychology, 7(743), 10.3389/fpsyg.2016.00743.

Cheng, T. (2019). On the very idea of a tactile field, or: A plea for skin-space. In Cheng, T., Deroy, O., \& Spence, C., (Eds.), Spatial senses: Philosophy of perception in an age of science (pp. 226-247). New York: Routledge.

Cheng, T. (2020). Molyneux's question and somatosensory spaces. In Ferretti, G., \& Glenney, B., (Eds.), Molyneux's question and the history of philosophy (pp. 300-311). London/New York: Routledge.

Cheng, T., \& Haggard, P. (2018). The recurrent model of bodily spatial phenomenology. Journal of Consciousness Studies, 25(3-4), 55-70.

Calzolari, E., Azañón, E., Danvers, M., Vallar, G., \& Longo, M. R. (2017). Adaptation aftereffects reveal that tactile distance is a basic somatosensory feature. Proceedings of the National Academy of Sciences of the United States of America, 114(17), 4555-4560.

Carruthers, G. (2008). Types of body representation and the sense of embodiment. Consciousness and Cognition, 17(4), 1302-1316.

Clark, A. (2004). Feature-placing and proto-objects. Philosophical Psychology, 17(4), 443469.

Corns, J. (2014). The inadequacy of unitary characterizations of pain. Philosophical Studies, 169(3), 355-378.

Corns, J. (2018). Recent work on pain. Analysis, 78(4), 737-753.

Cutter B., \& Tye, M. (2011). Tracking representationalism and the painfulness of pain. Philosophical Issues, 21(1), 90-109.

De Paepe, A. L., Crombez, G., Spence, C., \& Legrain, V. (2014). Mapping nociceptive stimuli in a peripersonal frame of reference: Evidence from a temporal order judgment task. Neuropsychologia, 56, 219-228. 
Fardo, F., Beck, B., Cheng, T., \& Haggard, P. (2018). A mechanism for spatial perception on human skin. Cognition, 178, 236-243.

Ferentzi, E., Bogdány, T., Szabolcs, Z., Csala, B., Horváth, Á., \& Köteles, F. (2018). Multichannel investigation of interoception: Sensitivity is not a generalizable feature. Frontiers is Human Neuroscience, 12(223), 10.3389/fnhum.2018.00223.

Ferrè, E., R., Iannetti, G. D., van Dijk, J. A., \& Haggard, P. (2018) Ineffectiveness of tactile gating shows cortical basis of nociceptive signaling in the thermal grill illusion. Scientific Reports, 8(1), 65-84.

Fulkerson, M. (2011). The unity of haptic touch. Philosophical Psychology, 24(4), 493-516.

Gallace, A., Torta, D. M. E., Moseley, G. L., \& Iannetti, G. D. (2011). The analgesic effect of crossing the arms. Pain, 152(6), 1418-1423.

Gozzano, S. (2019). Locating and representing pain. Philosophical Investigations, 42(4), 313332.

Gray, R. (2014). Pain, perception and the sensory modalities: Revisiting the intensive theory. Review of Philosophy and Psychology, 5, 87-101.

Gray, R. (2018). On the content and character of pain experience. Pacific Philosophical Quarterly, 100(1), 47-68.

Haggard, P., Cheng, T., Beck, B., \& Fardo, F. (2017). Spatial perception and the sense of touch. In de Vignemont, F. \& Alsmith, A. J. T., (Eds.), The subject's matter: Selfconsciousness and the body (pp. 97-114). Cambridge, MA: The MIT Press.

Haggard, P., \& Giovagnoli, G. (2011). Spatial patterns in tactile perception: Is there a tactile field?. Acta Psychologica, 137(1), 65-75.

Haggard, P., Iannetti, G. D., \& Longo, M. R. (2013). Spatial sensory organization and body representation in pain perception. Current Biology, 23(4), R164-R176. 
Head, H., \& Holmes, G. (1911). Sensory disturbances from cerebral lesions. Brain, 34(2-3), $102-254$.

Helm, B. W. (2002). Felt evaluations: A theory of pleasure and pain. American Philosophical Quarterly, 39(1), 13-30.

Hill, C. S. (2004). Ouch! An essay on pain. In Gennaro, R. J., (Ed.), Higher-order theories of consciousness. An anthology (pp. 339-362). Amsterdam/Philadelphia: John Benjamins Publishing Company.

Hochstetter, G. (2016). Attention in bodily awareness. Synthese, 193(12), 3819-3842.

Holly, W. J. (1986). The spatial coordinates of pain. The Philosophical Quarterly, 36(144), 343-356.

Hyman, J. (2003). Pains and places. Philosophy, 78(1), 5-24.

Klein, C. (2012). Imperatives, phantom pains, and hallucination by presupposition. Philosophical Psychology, 25(6), 917-928.

Knight, F. L. C., Longo, M. R., \& Bremner, A. J. (2014). Categorical perception of tactile distance. Cognition, 131(2), 254-262.

Koltzenburg, M., Handwerker, H. O., \& Torebjörk, H. E. (1993). The ability of humans to localise noxious stimuli. Neuroscience Letters, 150(2), 219-222.

Leder, D. (2016). The experiential paradoxes of pain. The Journal of Medicine and Philosophy: A Forum for Bioethics and Philosophy of Medicine, 41(5), 444-460.

Lederman, S. J., \& Klatzky (2009). Haptic perception: A tutorial. Attention, Perception, \& Psychophysics, 71(7), 1439-1459.

Longo, M. R. (2010). Implicit and explicit body representations. European Psychologist, 20(1), 6-15. 
Longo, M. R., \& Golubova, O. (2017). Mapping the internal geometry of tactile space. Journal of Experimental Psychology: Human Perception and Performance, 43(10), 18151827.

Longo, M. R., \& Haggard, P. (2010). An implicit body representation underlying human position sense. Proceedings of the National Academy of Sciences of the United States of America, 107(26), 11727-11732.

Mac Cumhaill, C. (2015). Perceiving immaterial paths. Philosophy and Phenomenological Research, 90(3), 687-715.

Mancini, F., Bauleo, A., Cole, J., Lui, F., Porro, C. A., Haggard, P., \& Iannetti, G, D. (2014). Whole-body mapping of spatial acuity for pain and touch. Annals of Neurology, 75(6), 917924.

Mancini, F., Haggard, P., Iannetti, G. D., Longo, M. R., \& Sereno, M. I. (2012). Fine-grained nociceptive maps in primary somatosensory cortex. The Journal of Neuroscience, 32(48), $17155-17162$.

Mancini, F., Sambo, C. F., Ramirez, J. D., Bennett, D. L. H., Haggard, P., \& Iannetti, G. D. (2013). Fovea for pain at the fingertips. Current Biology, 23(6), 496-500.

Mancini, F., Steinitz, H., Steckelmacher, J., Iannetti, G. D., \& Haggard, P. (2015). Poor judgment of distance between nociceptive stimuli. Cognition, 143, 41-47.

Marotta, A., Ferrè, E. R., \& Haggard, P. (2015). Transforming the thermal grill effect by crossing the fingers. Current Biology, 25(8), 1069-1073.

Martin, M. (1993). Sense modalities and spatial properties. In Eilan, N., McCarthy, R., \& Brewer, B., (Eds.), Spatial representation: Problems in philosophy and psychology (pp. 206218). Oxford: Oxford University Press.

Mattens, F. (2016). The sense of touch: From tactility to tactual probing. Australasian Journal of Philosophy, 95(4), 688-701. 
Matthen, M. (forthcoming). The dual structure of touch: The body vs peripersonal space. In F. de Vignemont (Ed.), The world at our fingertips. Oxford: Oxford University Press

Millar, B. (2019). Smelling objects. Synthese, 196, 4279-4303.

Moore, C. E. G., \& Schady, W. (1995). Cutaneous localisation of laser induced pain in humans. Neuroscience Letters, 193(3), 208-210.

Nelkin, N. (1994). Reconsidering pain. Philosophical Psychology, 7(3), 325-343.

Nudds, M. (2009). Sounds and space. In Nudds, M., \& O’Callaghan, C., (Eds.), Sounds and perception. New philosophical essays (pp. 69-96). Oxford: Oxford University Press.

O'Callaghan, C. (2008). Object perception: Vision and audition. Philosophy Compass, 3(4), 803-829.

O'Shaughnessy, B. (2009). The location of a perceived sound. In Nudds, M., \& O'Callaghan, C., (Eds.), Sounds and perception. New philosophical essays (pp. 111-125). Oxford: Oxford University Press.

O’Sullivan, B., \& Schroer, R. (2012). Painful reasons: representationalism as a theory of pain. The Philosophical Quarterly, 62(249), 737-758.

Phillips, I. (2013). Hearing and hallucinating silence. In Macpherson, F., \& Platchias, D., (Eds.), Hallucination. Philosophy and psychology (pp. 333-359). Cambridge, MA: The MIT Press.

Price, D. D., \& Aydede, M. (2005). The experimental use of introspection in the scientific study of pain and its integration with third-person methodologies: The experientialphenomenological approach. In Aydede, M. (Ed.), Pain. New essays on its nature and the methodology of its study (pp. 243-274). Cambridge, MA: The MIT Press.

Proske, U., \& Gandevia, S. C. (2012). The proprioceptive senses: Their roles in signaling body shape, body position and movement, and muscle force. Physiological Review, 92(4), $1651-1697$. 
Rachels, S. (2000). Is unpleasantness intrinsic to unpleasant experiences?. Philosophical Studies, 99(2), 187-210.

Ratcliffe, M. (2008). Touch and situatedness. International Journal of Philosophical Studies, 16(3), 299-322.

Reuter, K. (2017). The developmental challenge to the paradox of pain. Erkenntnis, 82(2), $265-283$.

Richardson, L. (2010). Seeing empty space. European Journal of Philosophy, 18(2), 227-243. Richardson, L. (2011). Bodily sensation and tactile perception. Philosophy and Phenomenological Research, 86(1), 134-154.

Richardson, L. (2013). Sniffing and smelling. Philosophical Studies, 162(2), 409-419.

Schlereth, T., Magerl, W., \& Treede, R.-D. (2001). Spatial discrimination thresholds for pain and touch in human hairy skin. Pain, 92(1-2), 187-194.

Scott, M. (2001). Tactual perception. Australasian Journal of Philosophy, 79(2), 149-160.

Smith, A. D. (2011). Tastes, temperatures, and pains. In Macpherson, F. (Ed.), The senses: Classical and contemporary philosophical perspectives (pp. 341-354). Oxford: Oxford University Press.

Soteriou, M. (2011). The perception of absence, space, and time. In Roessler, J., Lerman, H. \& Eilan, N., (Eds.), Perception, causation, and objectivity (pp. 181-206). Oxford: Oxford University Press.

Svensson, P., \& Arendt-Nielsen, L. (1995). Induction and assessment of experimental muscle pain. Journal of Electromyography and Kinesiology, 5(3), 131-140.

Tye, M. (2002). On the location of a pain. Analysis, 62(274), 150-153.

de Vignemont, F. (2010). Body schema and body image-Pros and cons. Neuropsychologia, 48(3), 669-680. 
de Vignemont, F. (2014). A multimodal conception of bodily awareness. Mind, 123(492), 889-1020.

de Vignemont, F. (2017). Pain and touch. The Monist, 100(4), 465-477.

de Vignemont, F., \& Jacob, P. (2012). What is it like to feel another's pain?. Philosophy of Science, 79(2), 295-316.

de Vignemont, F., Majid, A., Jola, C., \& Haggard, P. (2009). Segmenting the body into parts: Evidence from biases in tactile perception. The Quarterly Journal of Experimental Psychology, 62(3), 500-512.

Young, B. D. (2020). Perceiving smellscapes. Pacific Philosophical Quarterly, 101(2), 203223.

Young, N. (2017). Hearing spaces. Australasian Journal of Philosophy, 95(2), 242-255. 